\title{
ORQUIDEAS MEXICANAS...
}

Extractó de la conferencia que sustentó el Sr. Prof. Juan Balme en una reciente junta de nuestra Sociedad. El autor ilustró su plática con numerosas vistas a colores que proporcionó el Sr. G. Davis.

En vista del gran interés que han despertado en nuestra Sociedad las Orquideas, motivando la creación de una Sección especial que de preferencia se dedicará al estudio de las especies que tenemos silvestres en México, distribuidas en toda la zona intertropical, desde las costas hasta la Mesa Central, así como a promover, por medio de consejos y por conducto del Grupo Especial nombrado, su cultivo sobre bases racionales modernas, con esta fecha doy principio a una serie de pláticas ilustradas que sobre tan bellas plantas daré a Uds., contando para ello con la gran amabilidad de mi fino amigo y consocio Señor Ingeniero Glenn N. Davis, a quien renuevo mis agradecimientos por la gentileza que ha tenido en traer esta noche su proyector, pantalla y numerosas placas que de especies mexicanas fueron seleccionadas para hoy, las que, como están a colores, permitirán que sean fácilmente identificadas.

La familia de las orquídeas es una de las más extensas, y la mejor definida del reino vegetal, pasando ya de 15,000 las especies y variedades clasificadas.

Fácil es suponer que esa enorme cifra seguirá aumentando considerablemente cada año, ya sea por la introducción de especies y géneros nuevos, que se descubren en selvas hasta hoy inexploradas o por las que irán produciendo los hábiles sembradores, quienes, a medida que pasan los años, van logrando cada vez mejor éxito y nos presentan ya ejemplares que mucho se acercan a la perfección, destinados a enriquecer las numerosas colecciones existentes. 
Entre todos los Continentes, América es el más rico en Orquídeas epífitas, habiéndose encontrado en sus selvas, principalmente en las colombianas y venezolanas, las especies más hermosas, de las que algunas variedades, en los géneros CATTLEYA y ODONTOGLOSSUM, alcanzan precios verdaderamente fantásticos por lo elevado.

Según apuntes tomados de una lista aún incompleta de la Taxonomía de las Orquídeas Mexicanas, de la que es autor el bien conocido Profesor Don Casiano Conzatti, tan solo en México figuran 80 géneros, de los que se cuentan 250 especies, cantidad que de seguro, al agregársele las faltantes y sus numerosas variedades, pasará del doble.

De acuerdo con el lugar donde crecen, estas plantas herbáceas y perennes, reciben distintas denominaciones, siendo así como tenemos: Orquídeas terrestres, aquellas que viven en el suelo, mientras que las que viven sobre los árboles, o sobre las peñas revestidas de musgo, se les conoce por epifitas.

Las primeras, te hojas caducas, están provistas de tubérculos o de raices carnosas, mientras que las siguientes, de hojas perennes, unas veces se componen de tallos flexibles y delgados, semejando bejucos; otras veces tienen seudobulbos, más o menos voluminosos, donde almacenan sus reservas, soliendo tambićn tener, en vez de éstos, hojas o tallos carmosos.

Las especies epílitas, erróneamente llamadas "parásitas", tanto en el país, como en Centro y Sur América, aunque viven y se afianzan, para su crec miento, sobre los árboles, no deben ni pueden considerarse como tales, porque sólo aprovechan a aquéllos para sostenerse en el aire, por me lio de sus raíces adventicias y les piden únicamente apoyo y protección, pero no viven a sus expensas, como sucede con infinidad de plantas parásitas que, en el trópico, agobian y acaban por matar muy corpulentos árboles.

Sus raíces, por lo general abundantísimas y recubiertas de un tejido esponjoso, son de naturaleza distinta a las de la sespecies terrestres porque tienen que desempeñar dos funciones: una, haciendo el papel de ventosas, para afianzar las matas a la corteza, a fin de que puedan resistir a la acción de los vientos y la otra, para nutrirlas, absorbiendo, para ello, la hume lad así como reșiduos orgánicos y minúsculos hongos que encuentran en las rugosidades corticales.

$B$ en puede decirse que a esas plantas aéreas, que a veces viven a muy grandes alturas en los árboles y que parecen estar desprovistas de todo medio de existencia, les basta sólo ser acariciadas por la brisa 
húmeda que sopla gran parte del año en las zonas donde crecen, siendo un espectáculo verdaderamente digno de admiración, cuando se tiene el placer de recorrer aquellos bosques y cañadas, así como las márgenes de ríos, contemplar en lo más alto de las ramas, suspendidas en el vacio, grandes manojos de raíces colgantes, blancas y delgadas, que semejan gruesas cabelleras blancas, sobre las cuales se destacan, entre bulbos y hojas, largas guirnaldas de flores, de los coloridos más brillantes, maravillosamente bellas y que, por su gran abundancia, parecen nubes de mariposas o de insectos con alas desplegadas, cuya hermosura difícilmente puede imaginar quien no haya estado en aquellas fertilísimas zonas tropicales.

Por su modo peculiar de crecer, su aspecto general y sobre todo por la forma tan caprichosa de sus flores, lesde su aparición en Europa, que fuć a fines clel siglo XVIII, esas plantas tuvieron para el público, que podía contemplarlas en flor en los Establecimientos Cientificas y en las Exposiciones de Floricultura; un carácter misterioso, casi divino según ciertos autores, pues se decía que en ellas cl Creador esparciendo las formas más extrañas que pueda uno imaginarse, a las que agregó la quinta esencia de los perfumes más suaves ,había logrado obtener la más bella maravilla del reino vegetal.

Se hablaba de ellas, en voz baja y con cierto respeto y se les consideraba como de origen superior, pensándose, por los altos precios que al principio tuvieron algunas de ellas, que sólo estaba reservado a gentes millonarias el lujo de tenerlas, por ser las únicas capacitadas para mandarles construir elegantes casas de cristal, convenientemente acondicionadas, locales indispensables en aquellos países, para su conservación y cultivo, cosa que no sucede en nuestro México, donde el cultivo es sumamente sencillo y numerosas especies se acomodan perfectamente bicn al aire libre, en macetas, en huacales, sobre troncos de helechas y en los mismos árboles, si se tiene la precaución de protegerlas del frío durante los meses de invierno.

Esas plantas, por lo general productoras de flores de los más vivos colores, provistas de las formas más elegantes, exhalando los aromas más deliciosos, desde hace muchos años se han vuelto las favoritas de las clases privilegiadas y no puede concebirse fiesta solemne o banquete de renombre, sin que figuren en ellas canastillas de Orquideas, alternadas con otras de helechos de elegantes frondas, o de frutas de las más exquisitas. 
Ln resumen, las Orquideas son, en el mundo vegetal, la más granliosa expresión de la elegancia y de la fantasía, pues ellas adornan y perfuman los lugares donde viven, bastando sólo unas cuantas guirnaldas o flores de ellas, artísticamente distribuídas, para representar un espléndido rincón de la selva tropical.

Ahora si a todas sus cualidades, faltándome muchas por enumerar, agregamos las numerosas y bellas leyendas, que con sólo la evocación de su nombre se recuerdan, veremos que las orquídeas, por los sitios cleva los donde crecen, se colocan encima de la vulgaridad de las plantas terrestres, en el límpido azul del cielo, donde el espíritu maravillado al contemplarlas, saborea indécibles delicias.

Como nos lo mencionan en sus valiosas obras los más renombrados historiadores, el cultivo de ciertas especies de Orquídeas epífitas, estuvo muy en boga en esta, entonces lacustre ciudad de Tenochtitlán, así como en Tezcuco, en donde los Monarcas, Moctezuma Ilhuicamina y Netzahualcoyotl, por alición, se dedicaban ya a la Botánica, coleccionando plantas de las diversas regiones del Imperio Azteca, muchos años antes de que aquellas plantas fuesen conocidas en los Jardines Botánicos dol Viejo Mundo.

Entre las especies, porque no podía pasar inadvertida en aquella época, figuraba en primer lugar, por la caprichosa forma de sus flores y su exquisito aroma, la COATZONTECOMAXOCHITL, flor en forma de cabeza de víbora, que, juntamente con la TLILXOCHITL, flor negra, habían sido traídas al regreso de la campaña contra los totonacos, a mediados del siglo XV.

La primera aún muy buscada en la actualidad por los aficionados, corresponde a la STANHOPEA tigrina de Bateman, vulgarmente conocida con el nombre de TORITO y en cuanto a la segunda, con la que los totonacos pagaban sus tributos a Moctezuma, es aún cultivada por los mismos y corresponde a nuestra exquisita VAINILLA, la $V A N I L L A$ fragrans salisb, de fama mundial.

Numerosas otras especies de Orquídeas, conocidas por ZAUHTLI, que pertenecen a diversos géneros y especies, eran también muy apreciadas por los mismos mexicanos, quienes las utilizaban en sus industrias, en la medicina y como ornato, por la gran belleza de sus flores.

Por lo tanto, a México le corresponde el honor de haber sido el primer país del Mundo donde se haya hecho el cultivo de las Orquídeas epífitas, habiendo sido en los renombrados jardines Imperiales y 
Otra especic muy popular y bien conocida en los mercados capitalinos, es la "flor de Mayo", que corresponde a la LAELIA majalis o grandiflora, que aquí vemos con su varietad alba, la cual, traida en abundancia de los lugares donde crece silvestre se vende en las calles de la Capital en los meses de Mayo y Junio. Se le encuentra en lugares bastante cercanos y en extensas regiones del Bajío en donde aún persisten, para su dsignación, según zonas, los nombres indígenas de "Itzamaqua" y de "deuntza".

Extensamente distribuida sobre la vertiente del Pacífico y en regiones elevadas de los Estados del Sur, Guerrero, Oaxaca y Chiapas, la rubescens, conocida por "flor det Jesús", es muy apreciada, siéndolo más su variedad alba.

Varias son las especies de LYCASTE, siendo la más popular en la zona de Veracruz, la aromática, cuyas flores tienen un exquisito olor a canela, lo que ha originado que se le designe con el nombre vulgar de "canelita".

Su distribución, lo mismo que de la Deppei, es muy extensa sobre la vertiente del Golfo, llegando hasta Tabasco y Chiapas.

Entre las diversas especies, seguramente la más notable es la Skinneri, conocida en Chiapas y Guatemala con el nombre de "monjal", siendo su variedad alba, la "monja blanca" como así se le llama en aquel país, la que ha sido declarada flor nacional.

La MAXILLARIA praestans, de flores pequeñas, se encuentra distribuida sobre grandes extensiones en los Estados del Sureste.

Del género MORMODES, la especie pardinum y su variedad unicolor, que son las que se proyectan, son muy interesantes por la forma caprichosa de sus flores. Ambas se encuentran creciendo en zona tropical sobre la vertiente del pacífico, en los Estados de Oaxaca y Chiapas.

De regiones bastante elevadas y sobre ambas vertientes, son las diversas especies de ODONTOGLOSSUM mexicanas, figurando entre ellas:

La apterum o nebulosum, que crece sobre ambas vertientes, habiendo en sus variedades algunas notables, sobre todo en la Sierra Madre Occidental.

La Bictonense está también distribuida sobre ambas vertientes, habién tola de flores lilas con moreno y blanco con moreno también, las que son de gran efecto por el tamaño de sus espigas florales. 
Reales de Moctezuma y de Netzahualcoyotl, en donde por primera vez pudieron contemplarse, en el siglo XV.

Después de estas breves notas y con la valiosa ayuda de nuestro consocio Sr. Glenn Davis, pasaremos a las proyecciones, cuya distribución ha sido hecha por orden alfabético de géneros, siendo la primera:

La ACINETA Barkeri, conocida vulgarmente por boca de león, especie que se encuentra en extensas zonas tropicales de los Estados del Sur y del Sureste, preferentemente sobr la vertiente del Golfo.

Le siguen: la CATTLEYA citrina, extensamente distribuida sobre ambas vertientes, Golfo y Pacífico, de hermosas flores colgantes y muy ąromáticas, que los Aztecas conocían con el nombre de "coztictepexochitl" y que nuestros leñadores denominan aún "azucena amarilla del monte".

La CATTLEYA Skinneri, de los Estados del Sureste, conocida en Chiapas por "flor de San Sebastián", cuya distribución llega hasta Costa Rica, en donde es muy popular y se la conoce por "guaria".

La CHYSIS bractescens, que encontramos en extensa zona tropical, sobre la vertiente del Golfo, de hermosas y grandes flores, la que debido a la consistencia especial de las mismas, es designada "flor de cera".

Del género EPIDENDRUM, muy numerosas son las especies que tenemos y la ciliare, que se proyecta, crece en zona tropical, sobre ambas vertientes, en los Estados del Sur y del Sureste.

El Epidendrum cochleatum, vulgarmente conocido por "flor de concha", o "pulpito", debido a la forma caprichosa de sus flores, está extensamente distribuido en todos los Estados de la vertiente del Golfo, desde Tamaulipas hasta Campeche, habiendo sido esta la primera especie de Orquidea epífita que se introdujo en los cultivos europeos.

La especie Mariae, descubierta hace pocos años, en la Huasteca Veracruzana, figura entre las más bellas de este género,

El Epidendrum nemorale, conocida vulgarmente en Michoacán con el nombre de "trompillo", es también muy apreciada y el nombre que se le da es por sus seudobulbos que afectan la forma de un trompo.

En los Estados del Sur, especialmente sobre la vertiente del Pacífico, encontramos la especie ochraceum.

Una especie, cuyas flores de tallos colgantes son también de forma caprichosa, es la Parkinsorianum, o falcatum, extensamente distribuida 
en la zona tropical, sobre ambas vertientes, en los Estados del Sur y del Sureste, donde se la conoce con los nombres vulgares de "lirio de pata de gallo" o de "paloma".

El Epidendrum radiatum, conocida en Chiapas con el nombre vulgar de "flor de garrapata", se encuentra sobre ambas vertientes en los Estados del Sur y del Suroeste.

El Epidendrum virellinum, muy popular en zonas elevados de los Estados de Veracruz, Puebla y Oaxaca, en donde se le conoce con el nombre de "manuelitos", es otra de las especies que conocían los Aztecas y se les designaban con el nombre de "tonalxochitl".

La GONGORA galeata, vulgarmente conocida por "vaquita", crece en extensas zonas, tropical y subtropical de al vertiente del golfo, desde Veracruz hasta Tabasco.

Las diversas especies de GOVENIA eran también muy apreciadas por los Aztecas, quienes designaban a la liliacea, "petzacuxochitl" y a la superba, "cozticzacuxochitl". Ambas son conocidas hoy por "azucenas del monte".

Del género $L A E L I A$, extensamente distribuido desde las costas hasta la Mesa Central, en toda la extensa zona intertropical, son numerosísimas y bellas las variedades que de distintas especies tenemos, habiendo entre ellas unas de gran valor. Aquí tenemos la especie anceps, que, con su variedad alba, es la popular "flor de San Miguel" o "tzicxochitl", extensamente distribuida en regiones templadas de ambas vertientes, en los Estados de Veracruz, Puebla y Oaxaca.

La tan popular L. antumnalis, conocida por "flor de la calavera" o "de Todos Santos", que también era muy apreciada por los Aztecas y la nombraban "Chichiltictépetzacuxochitl". Aún se le conoce por "Ahoaxochitl" y "flor de encino", por ser sobre aquellos árboles donde crece de preferencia; se encuentra en regiones elevadas de nuestra Mesa Central, en los Estados de México, Morelos y Michoacán.

La L. furfuracea, crece también en regiones elevadas de la Sierra Madre Occidental, en los Estados del Sur, donde se la conoce por "flor de San Francisco".

La L. Gouldiana, que hasta ahora ha sido considerada como un híbrido natural entre autumnalis y anceps, es de las más hermosas entre las especies de guías largas. Crece en extensa zona del Estado de Veracruz, lindando con Hidalgo. Se le conoce con el nombre de "flor de muertos". 
La Cervantesii, conocida vulgarmente por "Jazmincito", que puede hallarse en los Estados de Veracruz, Morelos, México y Michoacán; la citrosmum, de hermosas y aromáticas flores, producidas en grandes racimos colgantes, que sobre la vertiente del Pacífico, en los Estados de México, Michoacán y Guerrero, recibe los nombres vulgares de "posolillo" y de "paracatachichi”. Fué esa especic, la que los insignes Botánicos La Llave y Lexarza, dedicaron a la memoria de Cuitláhuac, tenominándola CUITLAUZINA pendula.

De la cordatum, extensamente distribuida sobre ambas vertientes, desde los Estados de Veracruz y Michoacán hasta Chiapas, existen numerosas y bellas variedades, más o menos maculadas, pero la más hermosa especie, entre todas las de este género, es sin duda alguna la grande, cuyas bellas y grandes flores, se conocen en Norteamérica con el nombre de "baby orchid"; la "orquidea niño", por la forma especial de sus flores en cuyo centro se notan secciones que semejan un niño. Se la encuentra en el Estado de Ch:apas. Muy parecida a la anterior, pero de tamaño más chico, en los Estados de Michoacán y Guerrero, tenemos la Insleavi, que allá se conoce con el nombre de "terciopelo".

Procedente del Estado de Guerrero, la especie Londesboroughignum; es una de las más escasas, habiendo quedado muchos años sin volverse a encontrar. Cirece sobre la vertiente del Pacífico desde Michoacán hasta Oaxaca.

La Reichntieimi, que algunos autores clasifican leave, es también de la vert ente del Pacífico, encontrándose en los Estados de México, Michoacán y Gucrero. Produce largos tallos flores y sus flores poseen un exquisito aroma y en regiones elevadas de los Estados de Veracriz y Puebla, sobre la vertiente del Golfo hallamos la Rosii.

Del género ONCIDIUM encontramos especies en todas las zonas, cálida, templada y fria, sobre ambas vertientes, figurando entre ellas la incurom, que se conoce con el nombre de "angelitos". Igual densminación existe para la ornithorynchum, que también es designada "arañites", de las que las variedades albuin y sulphureum, son sumamente escasas.

El ORNITHOCEPHALUS iridifolius, ampliamente distribuido, se encuentra en zonas subtropicales de los Esta los del Sur y Sureste.

En cuanto a la hermosa SOBRALIA marcrantha, y sus numerosas variedades, conocidas vulgarmente por "lirio de San Antonio", se las encuentra en zonas tropicales de los Estados del Sur y del Sureste, sobre ambas vertientes, desde Veracruz y Michoacán hasta Chiapas, en- 
contrándose en ese último Estado la xantholeuca, también de gran hermosura.

Ahora llegamos al género $S T A N H O P E A$, del cual algunas especies eran grandemente apreciadas por los aztecas, quienes las denominaban "Coatzontecomaxochitl", que quiere decir "flor de cabeza de vibora". Actualmente se conocen por "toritos" y son extensamente distribuidas en nuestras zonas tropicales y templadas, llamando grandemente la atención por la forma muy caprichosa de sus flores y por el exquisito aroma que exhalan. La oculata, de manchas que semejan ojos y la tigrina, de colores más oscuros, fueron las más apreciadas y cultivadas en los jardines de Tenoxtitlán.

EI STENHORHYNCHUS cinnabarinus, conocido por "Cutzi" es una especie de Orquidea terrestre, de rico colorido anaranjado, que florece a principios de lluvias y la encontramos en extensas zonas elevadas. Cerca de la Capital se halla en terrenos contiguos a la carretera de Cuernavaca, en la sección del Marqués.

El STENORHYNCHUS cinnabarinus, conocido por "Cutzi" es rollados, extensamente distribuida sobre la vertiente del Golfo, en los Estados de Veracruz, Tabasco y Chiapas.

En la zona de la LAELIA autumnalis, en el Estado de Michoacán, donde puede apreciarse una gran mata de dicha especie.

La LAELIA Gouldiana, de flores sumamente renegridas, variedad extraordinaria se encurentra en el Estado de Hidalgo, en zona lindando con Veracruz.

Ahora son matorrales de ODONTOGLOSSUM citrosmum, que halló el Sr. Davis cerca de Valle de Bravo, Mex.

A inmediaciones de Veracruz, en compañía del ex-Presidente de la American Orchid Society, Sr. Wilcox Jones, quien nos visitó en ocasión del Congreso Internacional de Orquidófilos, el Sr. Davis tomó esta vista de Schomburgkia tibicinis.

Aquí doy por terminadas las breves notas que prepararé para esta noche, las cuales están limitadas a las hermosas placas que para proyecciones, sobre especies mexicanas, tiene nuestro buen amigo, Sr. Davis y que estas ilustraciones hayan servido para dar a ustedes una ligera idea de lo que son nuestras Orquideas, a quienes aún no las conocían, deseando sinceramente que muchos se animen a coleccionarlas y cultivarlas, pues hay entre ellas muchas especies sumamente rústicas, las que sólo requieren ser instaladas sobre árboles o en huacales rústicos, para producir hermosas flores, como habrán podido apreciarlo 
quienes hayan hecho una visita al Pueblo de Milpa Alta, D. F., en donde instalados sobre viejos paredones, pueden aún contemplarse en plena floración, con sus raices afianzadas a los adobes y a las piedras, grandes matorrales de LAELIA autumnalis. 